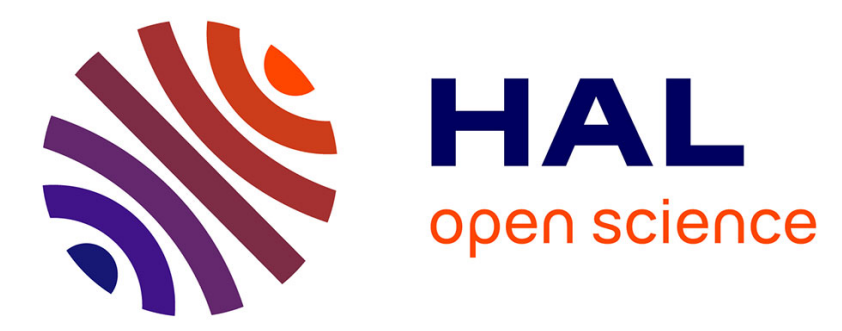

\title{
Convection flows driven by laser heating of a liquid layer
}

David Rivière, Bertrand Selva, Hamza Chraibi, Ulysse Delabre, Jean-Pierre

Delville

\section{To cite this version:}

David Rivière, Bertrand Selva, Hamza Chraibi, Ulysse Delabre, Jean-Pierre Delville. Convection flows driven by laser heating of a liquid layer. Physical Review E , 2016, 93 (2), pp.023112 (1-11). 10.1103/PhysRevE.93.023112 . hal-01343366

\section{HAL Id: hal-01343366 \\ https://hal.science/hal-01343366}

Submitted on 8 Jul 2016

HAL is a multi-disciplinary open access archive for the deposit and dissemination of scientific research documents, whether they are published or not. The documents may come from teaching and research institutions in France or abroad, or from public or private research centers.
L'archive ouverte pluridisciplinaire HAL, est destinée au dépôt et à la diffusion de documents scientifiques de niveau recherche, publiés ou non, émanant des établissements d'enseignement et de recherche français ou étrangers, des laboratoires publics ou privés.

\section{다(1)(2)}

Distributed under a Creative Commons Attribution - ShareAlikel 4.0 International 


\title{
Convection flows driven by laser heating of a liquid layer
}

\author{
David Rivière, Bertrand Selva, Hamza Chraibi, Ulysse Delabre, and Jean-Pierre Delville \\ University of Bordeaux, LOMA, UMR 5798, F-33400 Talence, France \\ and CNRS, LOMA, UMR 5798, F-33400 Talence, France
}

\begin{abstract}
When a fluid is heated by the absorption of a continuous laser wave, the fluid density decreases in the heated area. This induces a pressure gradient that generates internal motion of the fluid. Due to mass conservation, convection eddies emerge in the sample. To investigate these laser-driven bulk flows at the microscopic scale, we built a setup to perform temperature measurements with a fluorescent-sensitive dye on the one hand, and measured the flow pattern at different beam powers, using a particle image velocimetry technique on the other hand. Temperature measurements were also used in numerical simulations in order to compare predictions to the experimental velocity profiles. The combination of our numerical and experimental approaches allows a detailed description of the convection flows induced by the absorption of light, which reveals a transition between a thin and a thick liquid layer regime. This supports the basis of optothermal approaches for microfluidic applications.
\end{abstract}

\section{INTRODUCTION}

Since the pioneer work of Bénard and Rayleigh [1,2], natural convection flows induced by temperature gradients have been extensively studied [3] due to their presence in many natural phenomena. Such flows are also of major importance at intermediate and microscopic scales because temperature gradients are used, for instance, to control deposition of particles or solutes on substrates [4]. In general, temperature gradients can drive various effects such as: (i) natural convection resulting from the induced density gradient [5-7], (ii) particle transport by thermophoresis [8,9], or (iii) thermocapillary flows in samples with fluid interfaces $[10,11]$. Birikh was the first to study convective flows induced by longitudinal temperature gradients $[3,12]$ and his work has been recently extended to inclined temperature gradients [13]. However, at the micron scale, as in microfluidic applications, wellcontrolled temperature gradients may be difficult to induce by conventional techniques. In this context, laser heating via the absorption of the host fluid becomes an attractive route to induce such gradients. It is indeed a local, contactless, and versatile technique since the characteristics of the gradients can be controlled by the profile and the intensity of the laser beam. The timescale of the temperature rise induced by an infrared laser in a thin layer of water has proved to be of the order of a few milliseconds [14], which is small enough compared to low Reynolds hydrodynamics timescales and allows us to control almost instantaneously the temperature distribution in the sample. Recently, such laser heating has been exploited to induce thermocapillary migration in microchannels [15], to trap DNA by coupling convection to thermophoresis [8], or to induce the deposition of particles on solid substrates when the absorbing light is spatially modulated [16]. However, despite several studies on laser heating in fluids, to the best of our knowledge no study has yet described in details the key link between the induced temperature gradient and the resulting convective flows as a function of the laser parameters.

In the present investigation, we present both an experimental and a numerical work on natural convection driven by laser heating in a confined liquid layer and we characterize optically induced convective flows for future optothermal applications.
Interestingly, a global picture is presented to characterize the interplay between temperature profiles and hydrodynamics in terms of Peclet number and advection.

The paper is organized as follows: in Sec. II, we describe the experimental setup and the flow characterization techniques used in this work. Section III presents the hydrodynamic equations used in the numerical simulations. In Sec. IV, we compare experimental results with numerical predictions for three different liquid-layer thicknesses. Considering the nice observed agreement, we finally extend numerical predictions to other thicknesses and to unexplored experimental parameters to get a global characterization of the flows induced by laser heating in thin liquid layers.

\section{MATERIAL AND METHODS}

A sketch of the setup is presented in Fig. 1(a). A thin layer of aqueous solution is tightly confined between two microscope glass slides (thickness $1 \mathrm{~mm}$ ) using cover slips and mylar sheets as spacers. The solution is composed of Millipore water and tracers. The horizontal sizes of the water layer are $32 \times 25 \mathrm{~mm}$. Three layer thicknesses $H=185 \mu \mathrm{m}$, $H=310 \mu \mathrm{m}$, and $H=480 \mu \mathrm{m}$, very small compared to the horizontal extension, are investigated in this work. The sample is locally heated with a continuous infrared laser beam (LUMICS DSx1 laser diode, wavelength in vacuum $\lambda_{0}=1480 \mathrm{~nm}$ ), collimated by a doublet lens (THORLABS F810SMA), which is then focused with a beam waist $\omega_{0}=$ $10 \mu \mathrm{m}$ inside the sample with a microscope objective $O_{1}$ (ZEISS, NEOPLAN-NEOFLUAR $2.5 \times, \mathrm{NA}=0.075$ ). As the optical absorption of water is $\beta=2354 \mathrm{~m}^{-1}$ [14] at $1480 \mathrm{~nm}$, the laser beam heats the sample and induces a thermal gradient, causing a local variation of the density and setting the fluid in motion as sketched in Fig. 1(b). The absorbed power in the sample is defined by $P_{\text {abs }}=P_{\text {in }}[1-\exp (-\beta H)]$, where $P_{\text {in }}$ is the incident infrared laser power. Flows and temperature measurements are performed using epi-illumination (mercury lamp, $100 \mathrm{~W}$ ) with a long working distance objective $O_{2}$ (Olympus, $50 \times, \mathrm{NA}=0.5$ ) and frame grabbing with a $\mathrm{CCD}$ camera (Hamamatsu, C5405, $768 \times 576$ pixels, $25 \mathrm{fps}$ ). The objective $\mathrm{O}_{2}$ is controlled in the $z$ direction by a motorized 
(a)

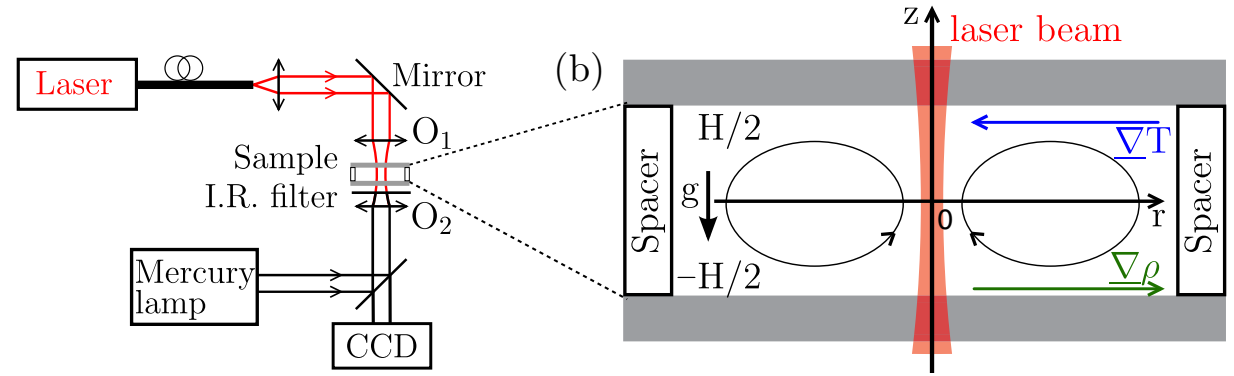

(c)



(d)
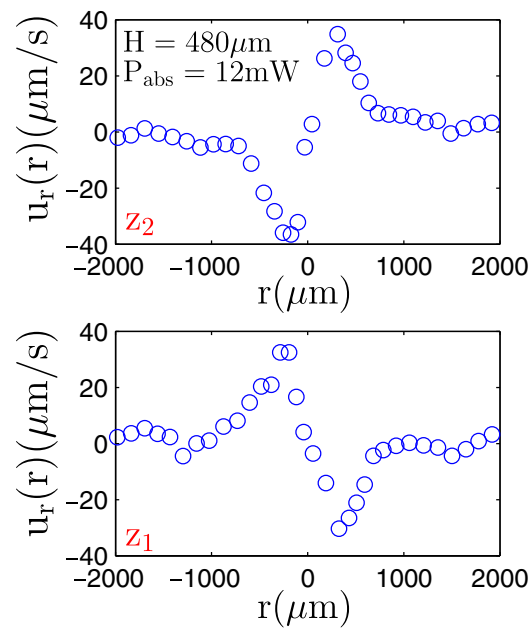

FIG. 1. (a) Sketch of the experimental setup, which shows the focusing, by a microscope objective $O_{1}$, of an infrared laser beam in the confined liquid. Images are collected with a microscope objective, $\mathrm{O}_{2}$, and a CCD camera. An infrared filter is placed below the sample to protect the camera against infrared light. (b) Zoom on the sample and schematic representation of the eddies induced by a density gradient $\underline{\nabla} \rho$ resulting from the temperature gradient $\underline{\nabla} T$ due to laser heating. The point of coordinates $(r=0, z=0)$ represents the middle of the sample (of height $H$ ) where the laser beam is focused. (c) Example of velocity profile $u_{r}(z)$ produced by the light-induced density gradient at $r=400 \mu \mathrm{m}$. (d) Corresponding velocity profiles $u_{r}(r)$ at $z=z_{1}$ and $z=z_{2}$, where $\left|u_{r}(z)\right|$ is the largest. $P_{\text {abs }}$ is the absorbed power that heats the liquid layer; the beam radius at $z=0$ is $\omega_{0}=10 \mu \mathrm{m}$.

stage (Marzhauser Wetzlar, scan IM $120 \times 80-1 \mathrm{~mm}$ ). This choice of long working distance objective is motivated by a desire for imaging the whole thickness of the sample. Finally, an infrared filter is placed in between the sample and the objective $\mathrm{O}_{2}$ to protect the CCD camera against infrared light.

\section{A. Flow visualization}

Velocity profiles inside the sample are measured using the particle image velocimetry technique (PIV) [17,18]. Fluorescent latex particles were used as tracers (Polysciences, Fluoresbrite plain YG 1.0 microns microspheres 9003-53-6) at a mass fraction $\Phi=0.5 \% \mathrm{wt}$ in solution. The setup used for the PIV measurements has been calibrated with Poiseuille flows in a microchannel. The details and the results of this calibration are given in Appendix 1. Typical velocity profiles induced by laser heating $u_{r}(z)$ and $u_{r}(r)$ are shown in Figs. 1(c) and 1(d), respectively, where velocities are taken as positive when flows move in the direction of the $r$ axis. Velocity profiles are obtained according to the following procedure: first, a $z$ scan is performed at an arbitrary radial distance from the beam axis (here at $r=400 \mu \mathrm{m}$ ). The velocity $u_{r}(z)$ profile is then plotted as shown in Fig. 1(c) where the positions $z_{1}$ and $z_{2}$ of the maximum of the velocity $u_{r}(z)$ are detected. These two altitudes are indicated by horizontal lines in Fig. 1(c). Then, horizontal scans on $r$ axis are performed at the altitudes $z_{1}$ and $z_{2}$ and give the velocity profiles $u_{r}(r)$ shown in Fig. 1(d). At a given $z$, it clearly appears that the velocity is antisymmetric and velocity profiles performed at $z_{1}$ and $z_{2}$ are antisymmetric with respect to the middle of the sample $(r=0, z=0)$, as seen in Fig. 1(d). These observations are consistent with the flow direction of eddies shown in Fig. 1(b) expected from the direction of the induced thermal gradient.

\section{B. Temperature measurement}

Temperature measurements were performed using a fluorescence thermometry technique $[14,19]$ with a different microscope objective $\mathrm{O}_{2}$ (Olympus, $2.5 \times, \mathrm{NA}=0.08$ ) for the visualization. We used rhodamine-B (RhB) (Sigma-Aldrich, $c=50 \mathrm{mg} / \mathrm{L}, \lambda_{\text {abs }}=555 \mathrm{~nm}, \lambda_{\text {em }}=580 \mathrm{~nm}$ ) in a carbonate buffer (HEPES at $c=50 \mathrm{mg} / \mathrm{L}$ ) as a temperature sensitive dye. The calibration of the fluorescence intensity is shown in Appendix 2. For a given temperature, the fluorescence intensity $I(T)$ of the $\mathrm{RhB}$ solution is measured by averaging 20 pictures. The normalized fluorescence intensity defined as $I(T) / I\left(T=20^{\circ} \mathrm{C}\right)$ shows a strong dependence on temperature, which is similar to the linear trend reported previously $(\approx-2 \% / \mathrm{K})[19]$. This technique implies that the temperature measured by fluorescence is the $z$-integrated temperature on 

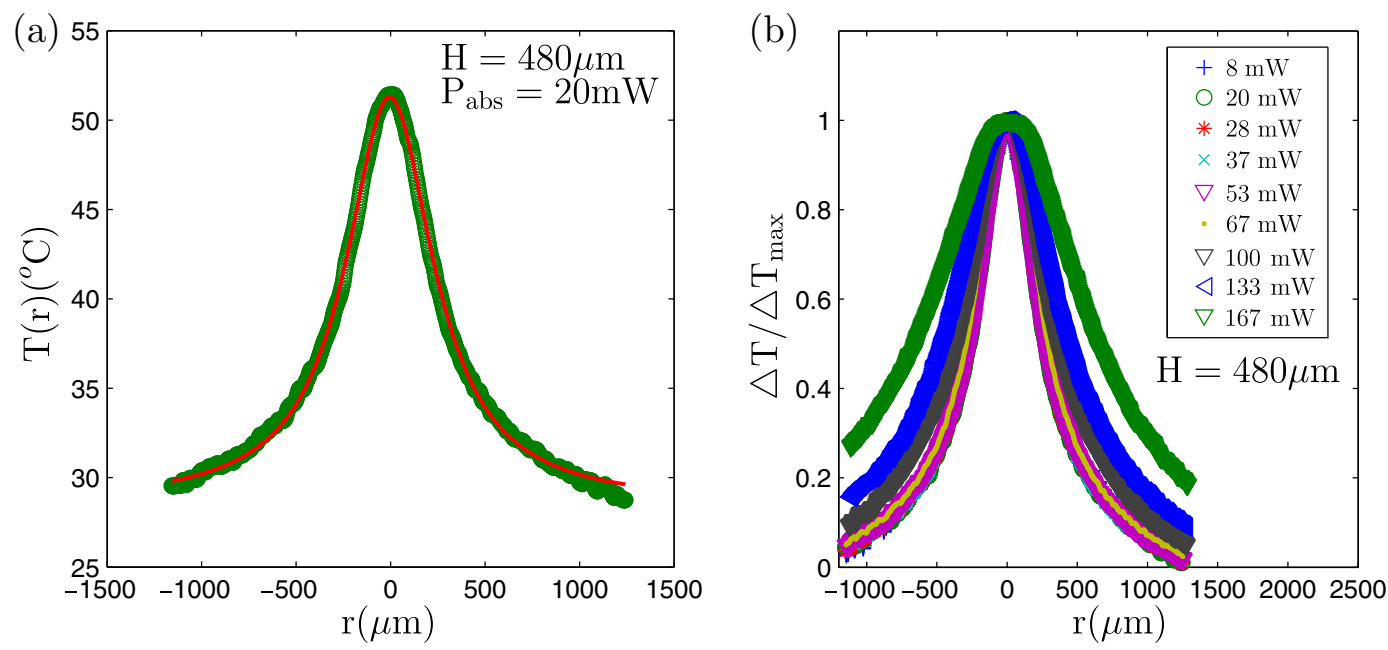

FIG. 2. (a) Typical temperature profile, here for $H=480 \mu \mathrm{m}$ and $P_{\text {abs }}=20 \mathrm{~mW}$, fitted by a Lorentzian function with a half width $\sigma=284.4 \mu \mathrm{m}$. (b) Overheating $\triangle T(r)$ normalized by the maximum temperature rise $\Delta T_{\max }$, for $H=480 \mu \mathrm{m}$ and for absorbed powers ranging from 8 to $167 \mathrm{~mW}$.

the thickness of the sample. This fluorescence measurement has been confirmed by a direct measurement of laser heating with a miniaturized thermocouple (Omega, type $K$, diameter $\simeq 50 \mu \mathrm{m}$ ) in pure Millipore water and presented in Appendix 3 . The good agreement between these two independent methods shows that Soret effect, i.e., thermophoresis of RhB in the solution due to laser heating [20], can totally be discarded in our experiment. A typical temperature profile induced by laser heating is shown in Fig. 2(a).

For the sake of simplicity, we have adjusted these temperature profiles with a Lorentzian fit of the form $T(r)=$ $\frac{\Delta T_{\max }}{1+\left(\frac{r}{\sigma}\right)^{2}}+T_{0}$ as suggested by several authors [8,14], where $\triangle T_{\max }=T(r=0)-T_{0}$ is the maximum temperature rise, $T_{0}$ is the room temperature, and $\sigma$ is the half width of the temperature distribution. As shown in Fig. 2(a), this fit captures well the radial variation of the $z$-integrated temperature in the sample. Temperature measurements for several absorbed powers $P_{\mathrm{abs}}$ are presented in Fig. 2(b) in which they have been normalized by $\triangle T_{\max }$. For $P_{\text {abs }}$ typically lower than 30 $\mathrm{mW}$, the half-width $\sigma$ remains almost constant. Conversely, above $30 \mathrm{~mW}, \sigma$ increases gradually with $P_{\text {abs }}$ for all the layer thicknesses investigated. We finally plotted in Figure 3, $\triangle T_{\max }$ and $\sigma$, as a function of the absorbed power for the three investigated thicknesses. As it appears in Figure 3(a), $\triangle T_{\max }$ is first linear with respect to the absorbed power up to 20-40 $\mathrm{mW}$ with mean slopes of $1.9 \pm 0.3^{\circ} \mathrm{C} / \mathrm{mW}$ for $H=185 \mu \mathrm{m}$, $1.5 \pm 0.2^{\circ} \mathrm{C} / \mathrm{mW}$ for $H=310 \mu \mathrm{m}$ and $1.6 \pm 0.2{ }^{\circ} \mathrm{C} / \mathrm{mW}$ for $H=480 \mu \mathrm{m}$, respectively. This linear behavior is detailed in Appendix 4. Above $\sim 70 \mathrm{~mW}$, the temperature rise starts to saturate. This transition between a low and a high power regime is confirmed by the fact that $\sigma$ appears to be almost constant for $P_{\mathrm{abs}} \leqslant 30 \mathrm{~mW}$ before increasing at higher power
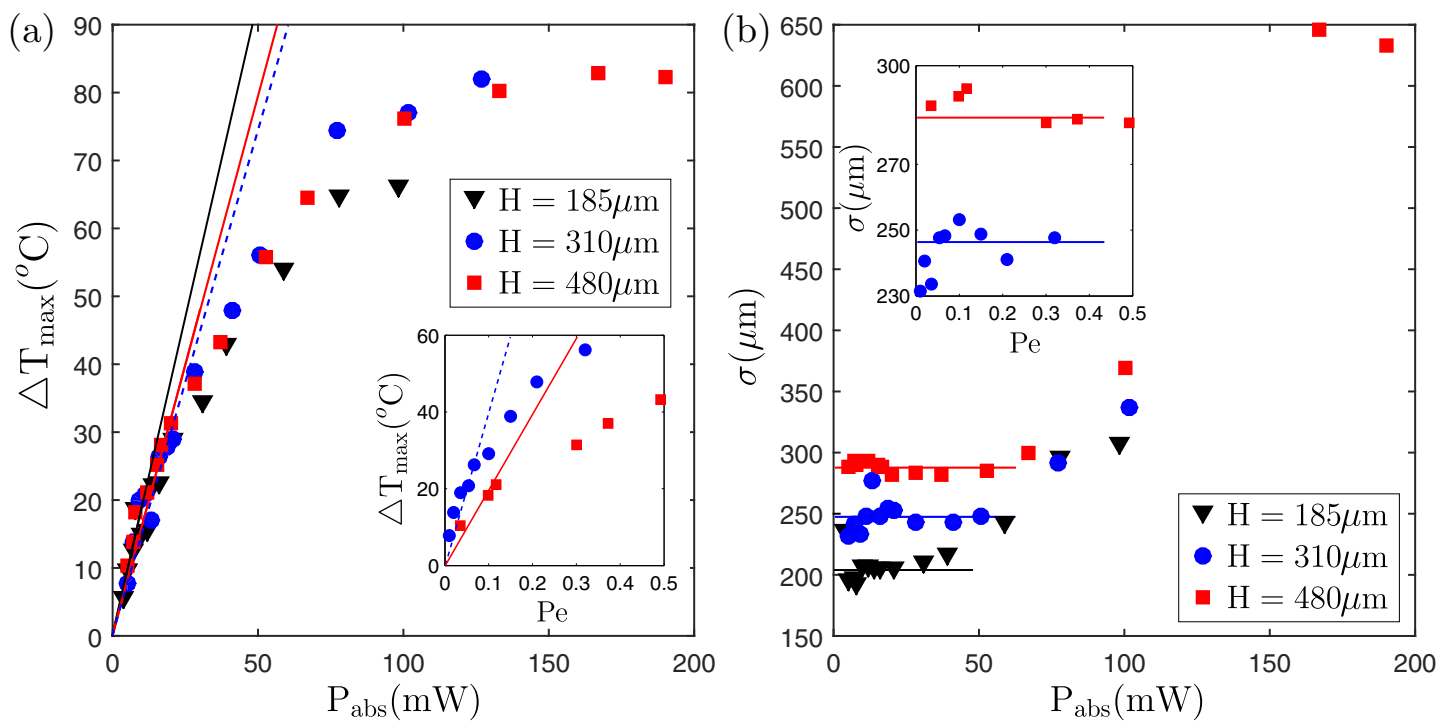

FIG. 3. Evolution of the maximum temperature rise $\Delta T_{\max }$ (a) and the temperature distribution width $\sigma$ (b) with the absorbed beam power. $\triangle T_{\max }$ (a) and $\sigma$ (b) are plotted in the insets as a function of the Peclet number for $H=310 \mu \mathrm{m}$ and $H=480 \mu \mathrm{m}$. 
[see Fig. 3(b)]. This behavior can be rationalized by estimating the magnitude of the temperature advection and diffusion in the heat equation given by:

$$
\frac{\partial T}{\partial t}+(\underline{u} \cdot \underline{\nabla}) T=\chi_{T} \underline{\nabla}^{2} T+\frac{\chi_{T}}{\Lambda} \beta I,
$$

where $\chi_{T} \approx 10^{-7} \mathrm{~m}^{2} \mathrm{~s}^{-1}$ and $\Lambda=0.596 \mathrm{Wm}^{-1} \mathrm{~K}^{-1}$ are, respectively, the thermal diffusivity and the thermal conductivity of water at $T=20^{\circ} \mathrm{C} ; \beta=2354 \mathrm{~m}^{-1}$ is the absorption of water at the used optical wavelength. $I(r, z)=\frac{2 P_{i n}}{\pi \omega_{0}^{2}} \exp \left[-\frac{2 r^{2}}{\omega_{0}^{2}}+\right.$ $\left.\beta\left(z-\frac{H}{2}\right)\right]$ is the intensity of the laser beam in the sample and $\omega_{0}$ the beam waist located at $z=0$. As twice the Rayleigh length $\frac{2 n \pi \omega_{0}^{2}}{\lambda_{0}} \sim 600 \mu \mathrm{m}$ is comparable to or larger than the height of the sample, we assumed a laser wave of cylindrical symmetry with a constant beam radius $\omega_{0} ; n$ is the index of refraction of water and $\omega_{0}=10 \mu \mathrm{m}$. Moreover, even if the absorption length, $\beta^{-1}=425 \mu \mathrm{m}$, is of the order of the thickness $H$, in a first approximation we do not take into account the axial beam intensity attenuation because our investigation attempts to describe laser convection in thin layers, as it is usually the case in microfluidics. In addition, as the characteristic thermal diffusion time is given by $\tau_{\text {th }}=H^{2} / \chi_{T} \sim 1 \mathrm{~s}$, a steady temperature profile is thus established after a few seconds in the sample. To get an order of magnitude of the thermal Peclet number Pe $=\|(\underline{u} \cdot \underline{\nabla}) T\| /\left\|\chi_{T} \underline{\nabla}^{2} T\right\| \sim U H / \chi_{T}$, which represents the ratio between advection of temperature and thermal diffusion, we chose the thickness of the sample $H$ as a characteristic length and the experimental characteristic velocity $U=u_{r, \max }$. For $H=310 \mu \mathrm{m}$ and $U=20 \mu \mathrm{m} \mathrm{s}^{-1}$ obtained at low powers $\left(P_{\text {abs }} \simeq 10 \mathrm{~mW}\right)$, we get $\mathrm{Pe}=0.09$. This value shows that advection can be neglected at low powers. This is experimentally confirmed in the insets of Fig. 3 where a transition between the diffusive to the advective regime emerges when $\mathrm{Pe} \sim 0.1-0.2$.

\section{HYDRODYNAMIC MODEL}

To describe the flow induced in the fluid layer by laser heating, we use the Stokes and mass conservation equations in stationary conditions:

$$
\eta_{0} \underline{\nabla}^{2} \cdot \underline{u}-\underline{\nabla} p+\Delta \rho(r) \underline{g}=\underline{0} ; \quad \underline{\nabla} \cdot \underline{u}=0,
$$

where $\underline{u}$ is the velocity field, $p$ the corrected pressure, $\triangle \rho(r) g=\left(\rho(T)-\rho_{0}\right) g$ the source term due to the density gradient with $\rho(T)$ and $\rho_{0}$ the density at temperature $T$ and $20^{\circ} \mathrm{C}$, respectively, and $\eta_{0}=10^{-3}$ Pa s the viscosity of water at $T=20^{\circ} \mathrm{C}$. Even though the viscosity is supposed to vary with temperature, for the sake of simplicity we consider $\eta_{0}$ as a constant to numerically resolve the flow field. The Reynolds number, defined as $\operatorname{Re} \sim \rho_{0} U H / \eta_{0}$, is $\operatorname{Re} \ll 1$, using the thickness $H$ and the velocity $U \approx 20 \mu \mathrm{m} / \mathrm{s}$ as characteristic experimental data; this justifies the use of Stokes equation. In addition, the mass conservation at steady state is given by $\underline{\nabla} \cdot[\rho(r) \underline{u}]=0$. As $\Delta \rho=\frac{\partial \rho}{\partial T} \Delta T \ll \rho_{0}$, the Boussinesq approximation still holds and leads to $\underline{\nabla} \cdot \underline{u}=0$ [21], implying that the fluid can still be treated as incompressible. Considering a Lorentzian temperature profile, we deduce the following expression for the source term:

$$
\Delta \rho(r) \underline{g}=\frac{\partial \rho}{\partial T} \Delta T \underline{g}=\frac{\rho_{0}|\alpha| \triangle T_{\max }}{1+\left(\frac{r}{\sigma}\right)^{2}} \underline{g},
$$

where $\alpha=-\frac{1}{\rho_{0}}\left(\frac{\partial \rho}{\partial T}\right)_{P} \approx 2.410^{-4} \mathrm{~K}^{-1}$ is the thermal expansion coefficient at $20^{\circ} \mathrm{C}$. Finally, we have neglected in Eq. (2) the density force $F_{b}$ resulting from the transfer of photon momentum to the fluid when photons are absorbed [22]. Indeed, the present investigation is devoted to large-scale free-convection flows, while the length scale associated to $F_{b}$, which is proportional to $I(r, z)$, is the beam waist $\omega_{0}$ $\left(\omega_{0} \ll H, \sigma\right)$. The velocity field must verify $\underline{u}=\underline{0}$ on all solid boundaries of the domain. In order to solve the system of Eqs. (2), we use a numerical procedure based on a boundary element method similar to that used by Chraibi et al. $[23,24]$ and adapted to a one-fluid problem. The characteristic width of the temperature field $\sigma$ is taken to be constant in simulations, $\sigma=200 \mu \mathrm{m}(H=185 \mu \mathrm{m}), \sigma=247 \mu \mathrm{m}(H=310 \mu \mathrm{m})$, and $\sigma=284 \mu \mathrm{m}(H=480 \mu \mathrm{m})$, which all correspond to experimental data in the linear regime of laser overheating [see Fig. 3(b)]. In order to generalize the comparisons, we also defined a reference velocity by balancing the viscous term with buoyancy such as $u_{0}=\frac{\omega_{0}^{2} \rho_{0} g|\alpha| \Delta T_{\max }}{\eta_{0}}$; here $\omega_{0}$ has been chosen as length scale as it is the natural size imposed by the laser. Finally, the dependence on the absorbed power is included in the maximum temperature rise from $\Delta T_{\max }=\gamma P_{\mathrm{abs}}$ in the linear regime [see Fig. 3(a)], which implies $u_{0}=\frac{\omega_{0}^{2} \rho_{0} g|\alpha|}{\eta_{0}} \gamma P_{\text {abs }}$.

\section{RESULTS AND DISCUSSION}

We present in this section a comparison between the experimental results and numerical simulations. In addition, we compute numerically the velocity profiles $u_{z}(r)$ in order to complete the description of hydrodynamic flows induced by laser heating.

\section{A. Comparison between experimental and numerical results}

We used a procedure that allows a direct comparison between the average value of $\gamma=\Delta T_{\max } / P_{\mathrm{abs}}$ found numerically and that measured experimentally. The comparison between experimental and numerical profiles is presented in Fig. 4, where we plotted the normalized velocity $u_{r} / u_{0}$ with respect to $z / H$ and $r / H$. A good agreement is observed between the shape of the numerical and experimental velocity profiles for the largest thicknesses; note that noise and fluctuations observed for the smallest thickness $(H=185 \mu \mathrm{m})$ are due to a relative decrease of the resolution related to the large depth of field of the long working distance of the used microcospe objective $\mathrm{O}_{2}$. We plotted in Fig. 5(a) the variation of the maximum radial velocity $u_{r, \max }$ with respect to the absorbed power $P_{\text {abs. }}$. In this figure, the continuous lines represent the numerical results of $u_{r, \max }=f\left(P_{\mathrm{abs}}\right)$. This shows that $u_{r \text { max }}$ varies linearly with the absorbed power when laser overheating is linear in $P_{\text {abs }}$; note that this trend supports our approximation, which consisted in neglecting the temperature dependence of viscosity at low absorbed powers. These experimental velocities allow us to calculate an experimental Peclet number as already shown in the insets of Fig. 3 in 

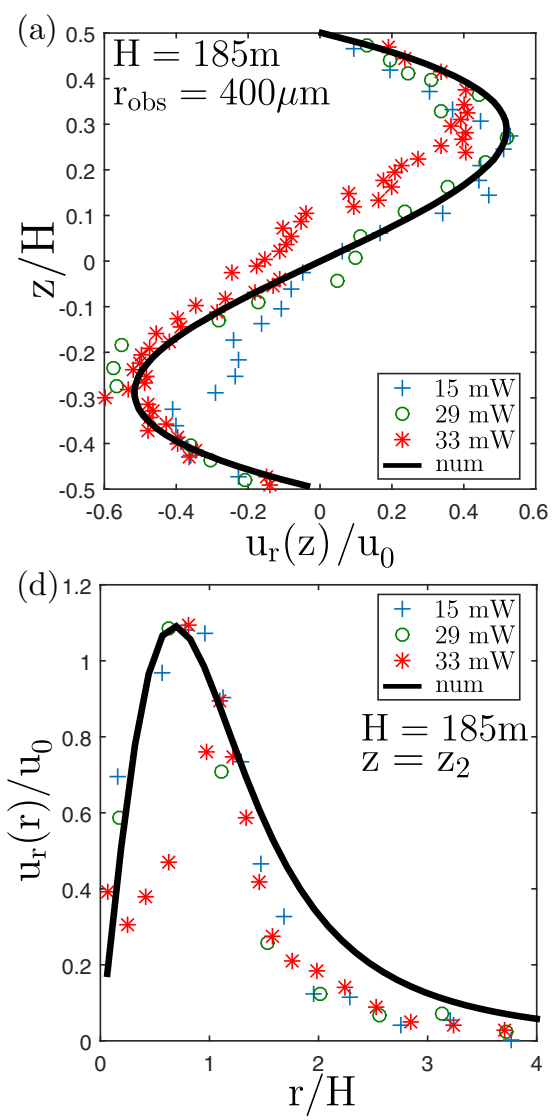
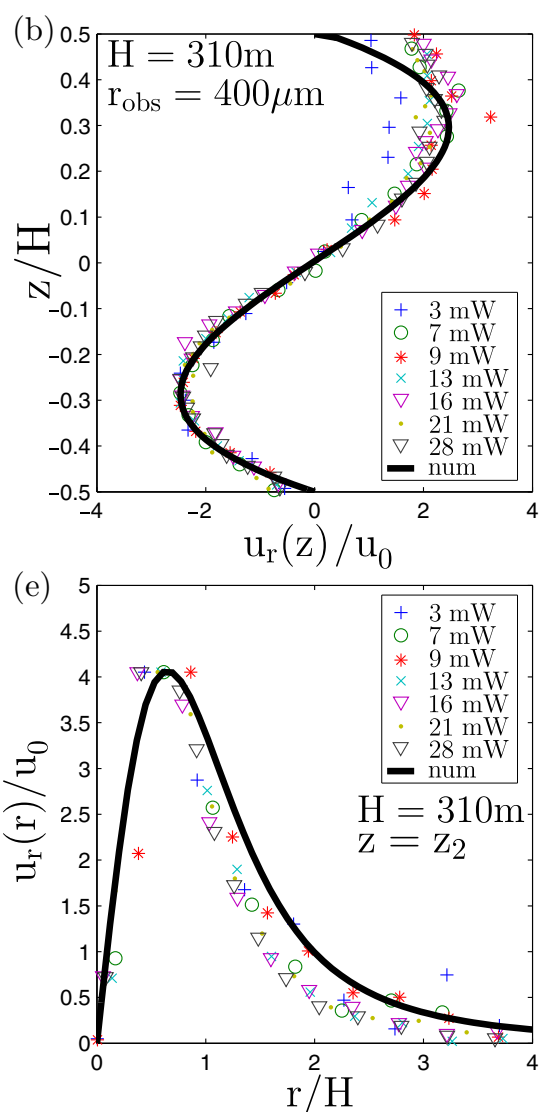

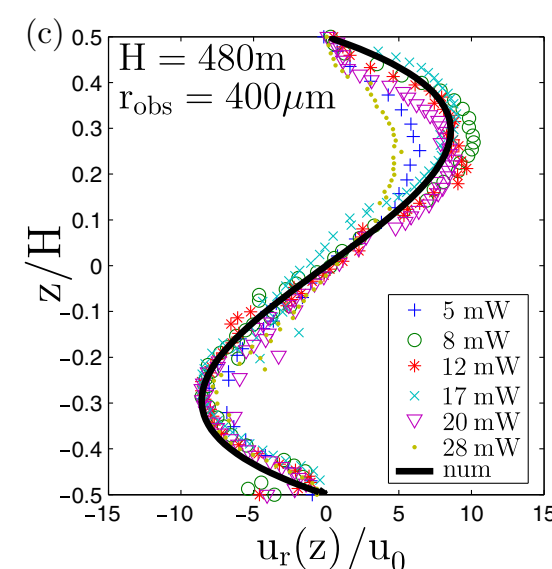

(f)

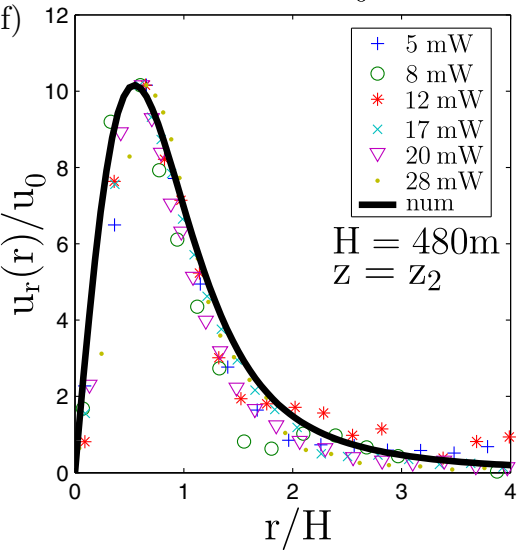

FIG. 4. Comparison between experimental results (symbols) and numerical simulations (line) for radial velocity profiles $u_{r}(z) / u_{0}(\mathrm{a})-(\mathrm{c})$ and $u_{r}(r) / u_{0}$ (d)-(f), respectively, for the thicknesses $H=185 \mu \mathrm{m}$ (a), (d), $H=310 \mu \mathrm{m}$ (b), (e), and $H=480 \mu \mathrm{m}$ (c), (f). The absorbed power ranges from 3 to $33 \mathrm{~mW}$.
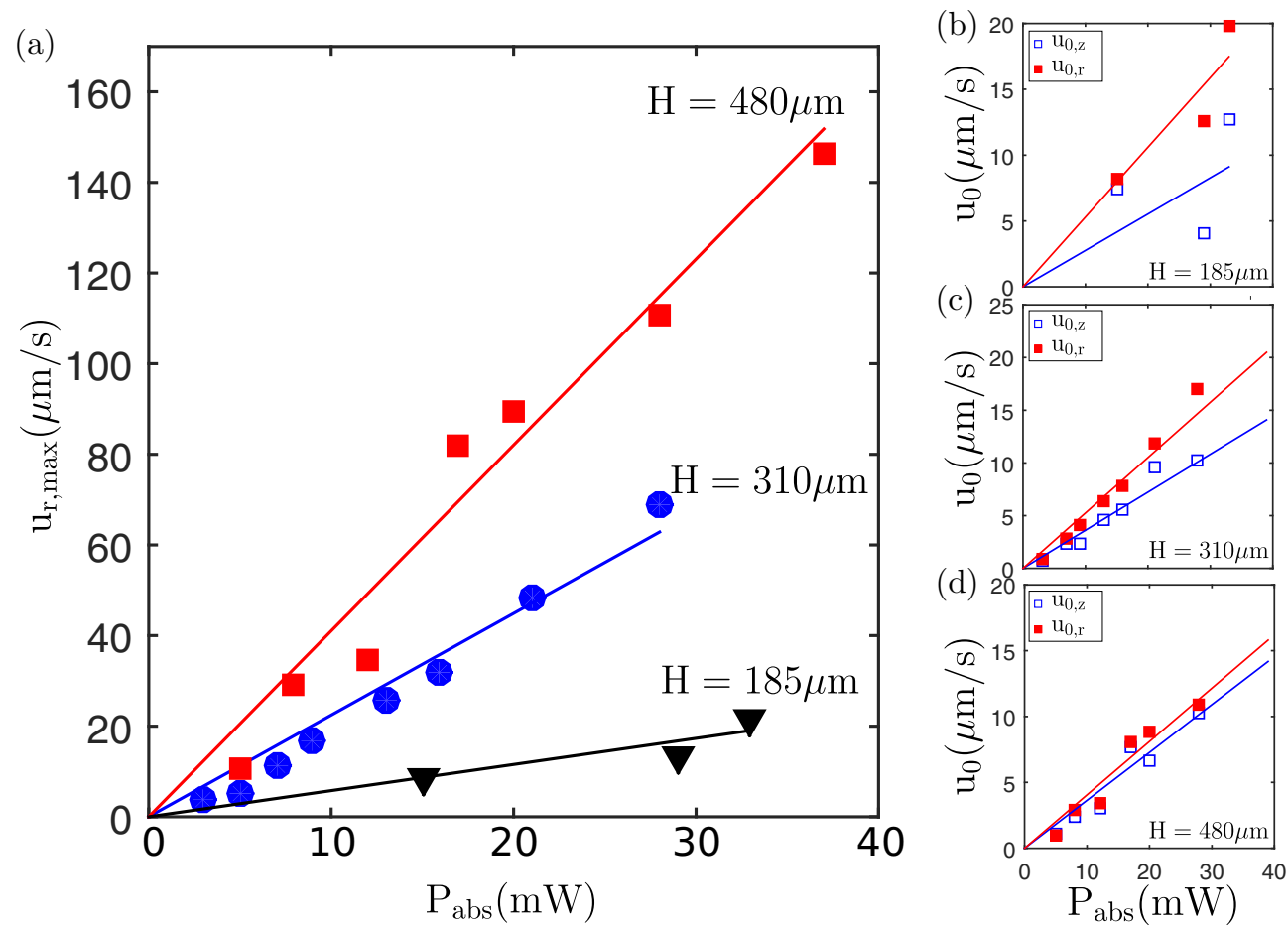

FIG. 5. (a) Comparison between experimental (symbols) and numerical (lines) results for the evolution of the maximum velocity $u_{r, \text { max }}$ with the absorbed power $P_{\text {abs }}$ for each sample height. Variation of the reference velocities $u_{0, z}$ and $u_{0, r}$ determined from $u_{r}(z)$ and $u_{r}(r)$, respectively, for $H=185 \mu \mathrm{m}$ (b), $H=310 \mu \mathrm{m}$ (c), and $H=480 \mu \mathrm{m}$ (d), with respect to the absorbed power (see text). 

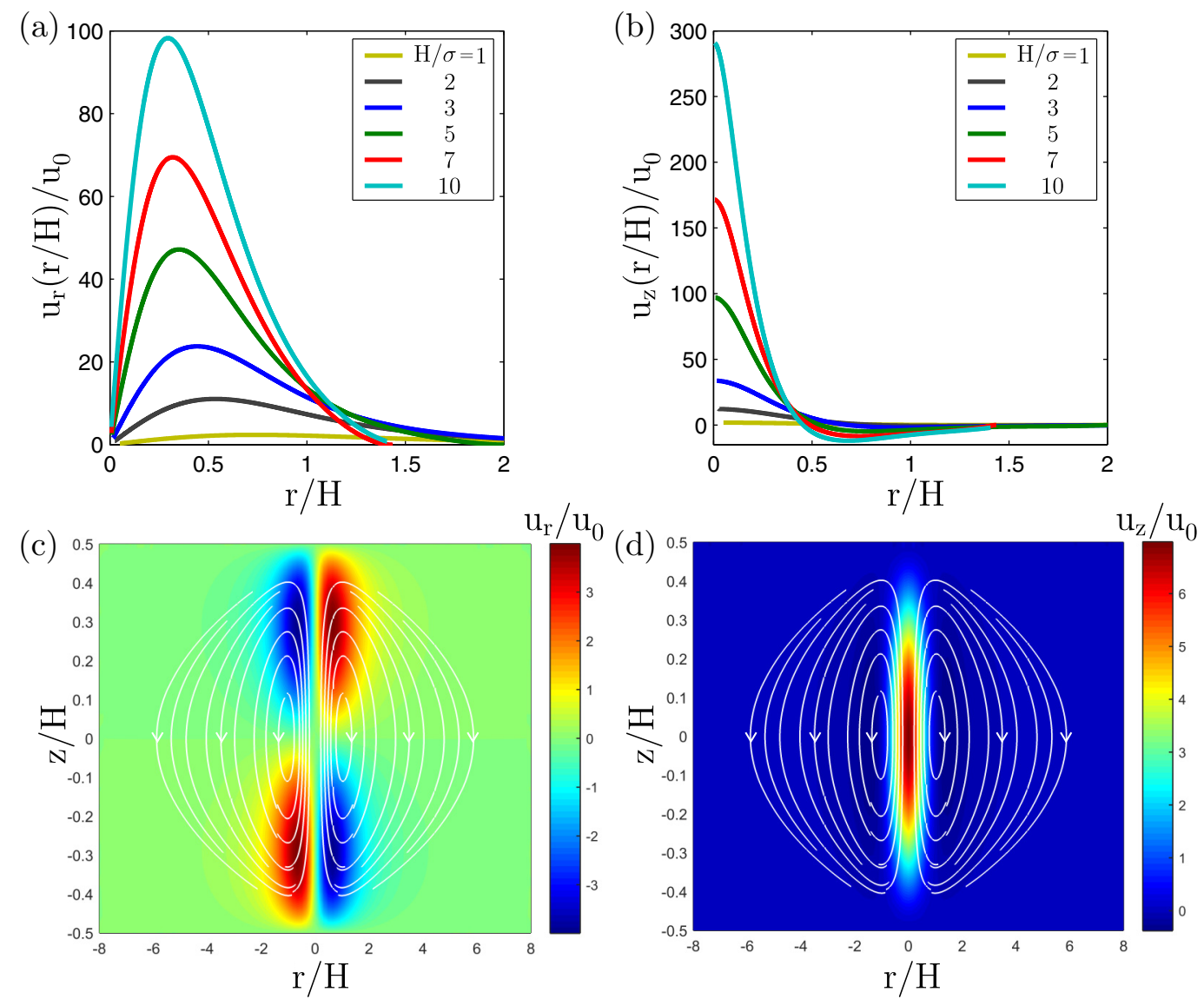

FIG. 6. Velocity profiles $u_{r}(r) / u_{0}$ (a) and $u_{z}(r) / u_{0}$ (b) at the altitude $z_{2}$ for several ratios $H / \sigma$, ranging from 1 to 8 , obtained by numerical simulations when fixing $\sigma=250 \mu \mathrm{m}$. (c) Thermal convection patterns and magnitude of $u_{r}(r) / u_{0}$ for $H / \sigma=1.24$, which corresponds to $H=310 \mu \mathrm{m}$. (d) Thermal convection patterns and magnitude of $u_{z}(r) / u_{0}$ for $H / \sigma=1.24$. Arrows indicate the flow direction.

order to identify a transition between the diffusive and the advective regime. To determine the slopes numerically, we first determined for each experimental velocity profile the value of $u_{0}$ giving the best agreement in amplitude with the numerical simulations for $u_{r}(z)$ and $u_{r}(r)$. Then, we plotted theses values of $u_{0}$ versus the absorbed power for $H=185 \mu \mathrm{m}$ in Fig. 5(b), $H=310 \mu \mathrm{m}$ in Fig. 5(c), and for $H=480 \mu \mathrm{m}$ in Fig. 5(d). We deduced the slope $u_{0} / P_{\text {abs }}$ and thus obtain the average temperature elevation coefficient $\gamma$. We found in average a temperature elevation $\Delta T_{\max } / P_{\text {abs }} \approx$ $2.3 \pm 0.3{ }^{\circ} \mathrm{C} / \mathrm{mW}$ for $H=185 \mu \mathrm{m}, \Delta T_{\max } / P_{\mathrm{abs}} \approx 1.8 \pm$ $0.3^{\circ} \mathrm{C} / \mathrm{mW}$ for $H=310 \mu \mathrm{m}$, and $\Delta T_{\max } / P_{\text {abs }} \approx 1.6 \pm$ $0.4^{\circ} \mathrm{C} / \mathrm{mW}$ for $H=480 \mu \mathrm{m}$, which are of the order of the values found experimentally, $\Delta T_{\max } / P_{\mathrm{abs}}=$ $1.9 \pm 0.3{ }^{\circ} \mathrm{C} / \mathrm{mW}, 1.5 \pm 0.1^{\circ} \mathrm{C} / \mathrm{mW}$, and $1.6 \pm 0.2^{\circ} \mathrm{C} / \mathrm{mW}$, respectively.

\section{B. Dependence of the flow on the characteristic lengths $H$ and $\sigma$ \\ 1. Scaling analysis}

In order to predict some general trends for $u_{r}$ and $u_{z}$, we consider the two asymptotic regimes: the thin-layer $(H / \sigma \ll$ 1) and the thick-layer $(H / \sigma \gg 1)$ regime. In the thin-layer regime $(H / \sigma \ll 1)$, one can use the classic Birikh profile [3] obtained in the case of a constant horizontal thermal gradient and in the lubrication approximation:

$$
u_{r}(r, z)=\frac{H^{3}}{12 \eta_{0}} g \frac{\partial \rho}{\partial r}\left[2\left(\frac{z}{H}\right)^{3}-3\left(\frac{z}{H}\right)^{2}+\left(\frac{z}{H}\right)\right] .
$$

As $H$ and $\sigma$ can be controlled independently, we will predict the dependence of $u_{r}$ and $u_{z}$ on the thickness $H$ alone (with $\sigma$ considered as a constant) and on $\sigma$ alone (with $H$ considered as a constant). First, $\sigma$ is considered as a constant to analyze the dependence of this equation on the thickness. From the classic Birikh profile [Eq. (4)] we deduce $u_{r} \sim H^{3}$ in the thin-layer regime $(H / \sigma \ll 1)$. Using the mass conservation $(\nabla \cdot u=0)$, we find $u_{r} / \sigma \sim u_{z} / H$, which gives $u_{z} \sim H^{4} / \sigma \sim H^{4}$ (for $H / \sigma \ll 1$ ). To consider the thick-layer regime $(H / \sigma \gg 1)$, we simplify the Stokes equation [Eq. (2)] and get $\eta\left(u_{r} / \sigma^{2}\right) \sim \frac{\partial p}{\partial r}$. By integration between $z=0$ and $z=H / 2$, we find $u_{r} \sim \frac{\partial \rho}{\partial r} g \sigma^{2} H$ since $p \sim \rho(r) g z$; we deduce $u_{r} \sim H \sigma \sim H$. Once again the mass conservation leads to $u_{z} \sim H^{2}$. A second analysis can be performed by fixing $H$ and investigating the effect of $\sigma$ on the amplitude of the velocity field. Equation (4) implies that $u_{r} \sim H^{3} \frac{\partial \rho}{\partial r}$ in the thin-layer regime. Hence, we obtain $u_{r} \sim \Delta \rho / \Delta r \sim \Delta T / \Delta r \sim 1 / \sigma$. Using the mass conservation, we find $u_{r} / \sigma \sim u_{z} / H$ and obtain $u_{z} \sim 1 / \sigma^{2}$. For the thick-layer regime, the integration of the simplified Stokes equation between $z=0$ and $z=H / 2$ leads to $u_{r} \sim \frac{\partial \rho}{\partial r} g \sigma^{2} H$, making use of $p \sim \rho(r) g z$. We deduce 

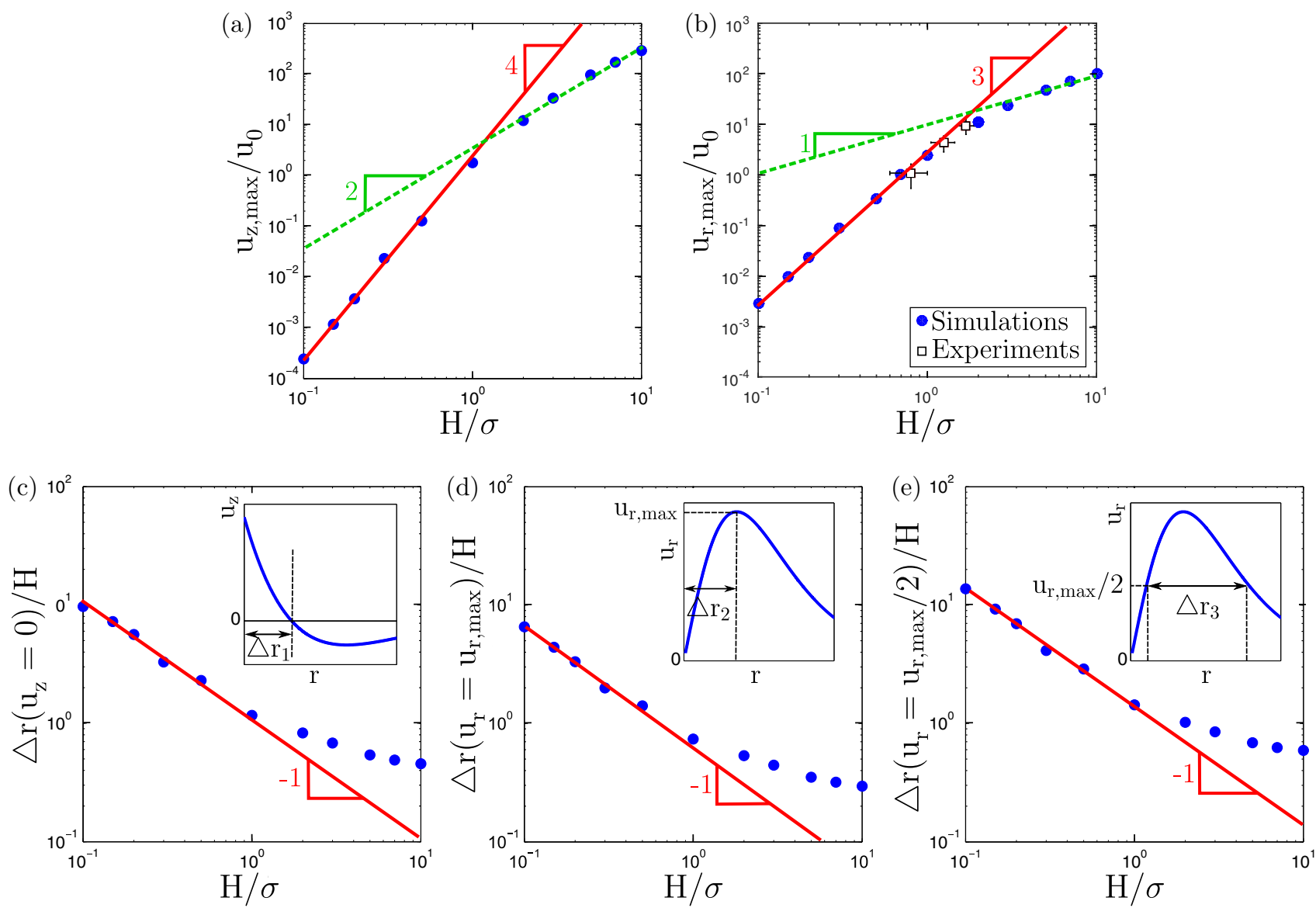

FIG. 7. (a) and (b) represent, respectively, the maximum axial and radial velocity with respect to $H / \sigma$ for $\sigma=250 \mu$ m. (b) shows a comparison between the simulations and the experimental data. Thicknesses are normalized by their corresponding $\sigma$. (c) $-(\mathrm{e})$ represent the evolution of the characteristic width $\Delta r$ of $u_{z}$ and $u_{r}$ with respect to $H / \sigma$, when considering the full width at $u_{z}=0$ (c), the abscissa of the maximum radial velocity (d), and the full width at half maximum (e), as indicated in the respective insets.

$u_{r} \sim H \sigma \sim 1 / \sigma^{-1}$, and mass conservation leads finally to $u_{z} \sim 1 / \sigma^{0}$. We then expect a transition between the thin and the thick layer regime when varying $\sigma$ or $H$.

Considering the agreement between experimental data and numerical simulations, we investigated these scaling laws numerically and analyzed the dependence of the flow magnitude to the thickness layer in the range $H / \sigma=0.1-10$. As previously, these simulations were performed by neglecting the advection in the thermal equation $(\mathrm{Pe}=0)$, a situation that corresponds to liquids with either large viscosities or high thermal diffusion coefficients.

\section{Simulation of the effect of the thickness $H$}

We present a parametric numerical investigation for both radial and axial velocities with $H / \sigma$ ranging from 0.1 to 10 and $\sigma$ set to $250 \mu \mathrm{m}$, which is of the same order of magnitude as the characteristic width of the temperature field determined experimentally. Figures 6(a) and 6(b) show the velocity profiles $u_{r}(r)$ and $u_{z}(r)$ as a function of $H / \sigma$. As expected, the maximum velocities increase with $H / \sigma$ for both radial and axial components. An example of the velocity fields and streamlines is shown in Figs. 6(c) and 6(d). In addition, we plotted in Figs. 7(a) and 7(b) the normalized maximum velocities $u_{z, \max }$ and $u_{r, \max }$ with respect to $H / \sigma$. Figures 7(a) and 7(b) show that the maximum velocities scale as $u_{z} \sim H^{4}$ and $u_{r} \sim H^{3}$ for $H / \sigma \ll 1$ and as $u_{z} \sim H^{2}$ and $u_{r} \sim H$ for $H / \sigma \gg 1$ ( $\sigma$ is constant in this numerical investigation). This confirms the scaling arguments presented previously for the effect of the thickness $H$. Note that we recover the $H$ variation of the vertical velocity found by Rusconi et al. [22] for thick layers in the context of thermophoresis induced by laser heating. We also calculate the experimental value of $u_{r, \max } / u_{0}$ for each thickness $H$ (normalized by its experimental value of $\sigma$ ) to present in Fig. 7(b) a comparison between the numerical simulations and our experimental data.

Finally, we investigated numerically the characteristic extension of the flow $\Delta r$, for $u_{z}$ and $u_{r}$. We use three definitions of $\Delta r$ defined in Figs. 7(c)-7(e), which show that the radial extension $\Delta r / H$ tends to scale as $(H / \sigma)^{-1}$ for $H / \sigma \ll 1$. This implies that the characteristic length $\Delta r$ of the flow is independent of the height $H$ of the fluid layer. For $H / \sigma>1$, $\triangle r$ results from an interplay between $H$ and $\sigma$ that shows a transition between the thin- and thick-layer regimes before a saturation, which predicts $\Delta r \sim H$ when $H / \sigma \gg 1$.

\section{Simulation of the effect of the thermal characteristic length $\sigma$}

Similarly, we investigated the radial and axial velocities with $H / \sigma$ ranging from 0.1 to 10 when $H$ is set to a constant 

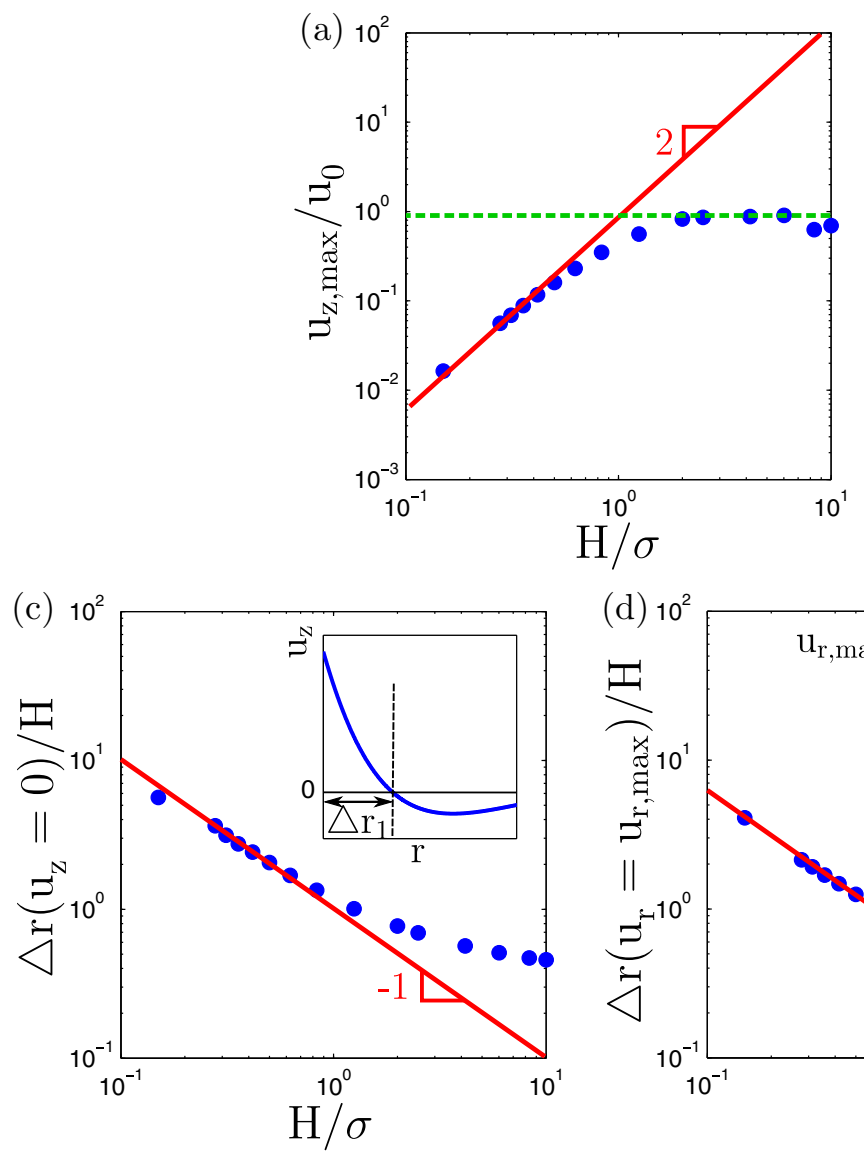
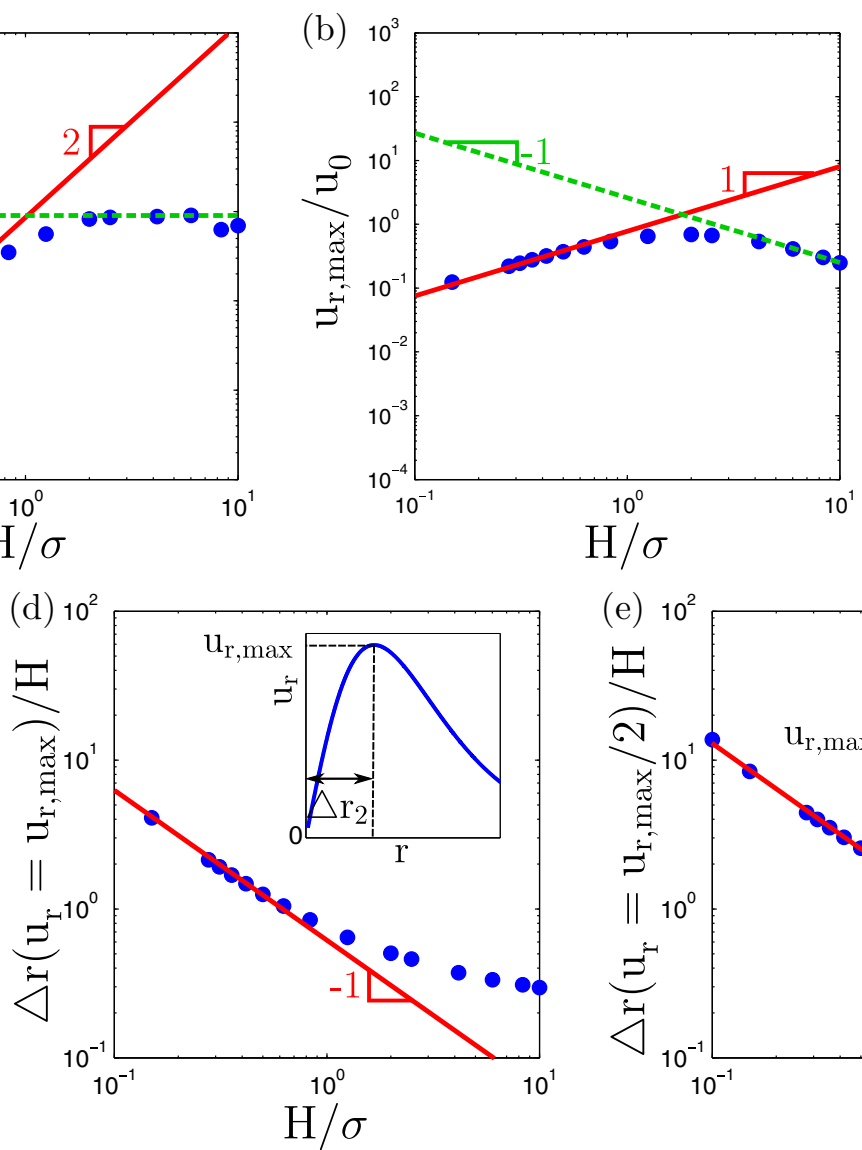

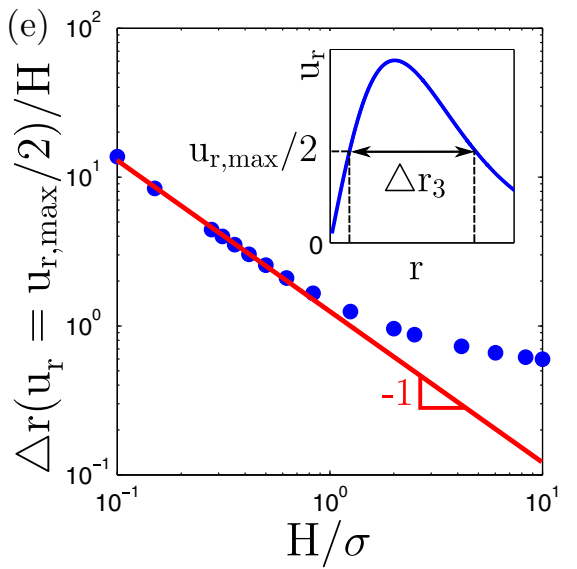

FIG. 8. (a) and (b) represent, respectively, the maximum axial and radial velocity with respect to $H / \sigma$ for $H$ constant. (c)-(e) represent the evolution of the characteristic width $\Delta r$ of $u_{z}$ and $u_{r}$ with respect to $H / \sigma$, when considering the full width at $u_{z}=0$ (c), the abscissa of the maximum radial velocity (d), and the full width at half maximum (e), as indicated in the respective insets.

( $H=125 \mu \mathrm{m}$ ). Figures 8(a) and 8(b) show the evolution of the maximum velocities as a function of $H / \sigma$. As indicated by the asymptotic lines, the arguments presented in the scaling analysis are verified, i.e., $u_{z} \sim 1 / \sigma^{2}$ and $u_{r} \sim 1 / \sigma$ for $H / \sigma \ll 1$ and $u_{z} \sim 1 / \sigma^{0}$ and $u_{r} \sim 1 / \sigma^{-1}$ for $H / \sigma \gg 1$. Figures 8(c)-8(e) also show that the characteristic length of the flow $\Delta r$ scales as $\sigma$ for $H / \sigma \ll 1$ and becomes independent of $\sigma$ for $H / \sigma \gg 1$. These results are important for flow control in microfluidic devices because the thickness of the channel is usually kept constant while the thermal extension can be tuned optically by beam-diameter variations.

\section{CONCLUSION}

In conclusion, we characterized experimentally and numerically the convection induced by the heating of a confined liquid layer of various thicknesses with an infrared laser beam. Using a fluorescence technique, we measured accurately the temperature profile to characterize in details the driving source of the hydrodynamic flows set in the sample. This enabled us to determine experimentally the transition between the diffusive and the advective regime where the temperature distribution starts to be coupled with convection. The comparison between the experimental and numerical velocity distributions showed that a simple model allows for a quantitative agreement. Based on this agreement, we extended numerical predictions to a large panel of sample thicknesses and temperature distribution. This parametric investigation revealed a transition between two regimes. A first regime specific to thin layers where the characteristic length of the flow is the radial extension of the thermal field and where the scaling of the velocity is in agreement with the classic Birikh profile. The second regime, describing thick layers, shows that the characteristic length of the flow strongly depends on the layer thickness while the velocity magnitude shows a slower increase with the film thickness. Finally, our numerical and experimental approaches allow for a detailed description of the convection flows induced by localized laser light and furnish the bases to understand many microfluidic experiments on particle manipulation by laser heating. More generally it opens a route on light-induced convection [25] and optothermal effects for optofluidic applications.

\section{ACKNOWLEDGMENTS}

Authors gratefully acknowledge the financial support from the Conseil Régional d'Aquitaine (MEMs Project No. 201211010190). Authors thank Jean-Baptiste Salmon for fruitful discussions and advice. We also thank Levi Haelman, William Benharbone, and Sebastien Cassagnere of the LOMA for their help in the technical realization of the setup and Julio Larios for his work on the calibrations. 

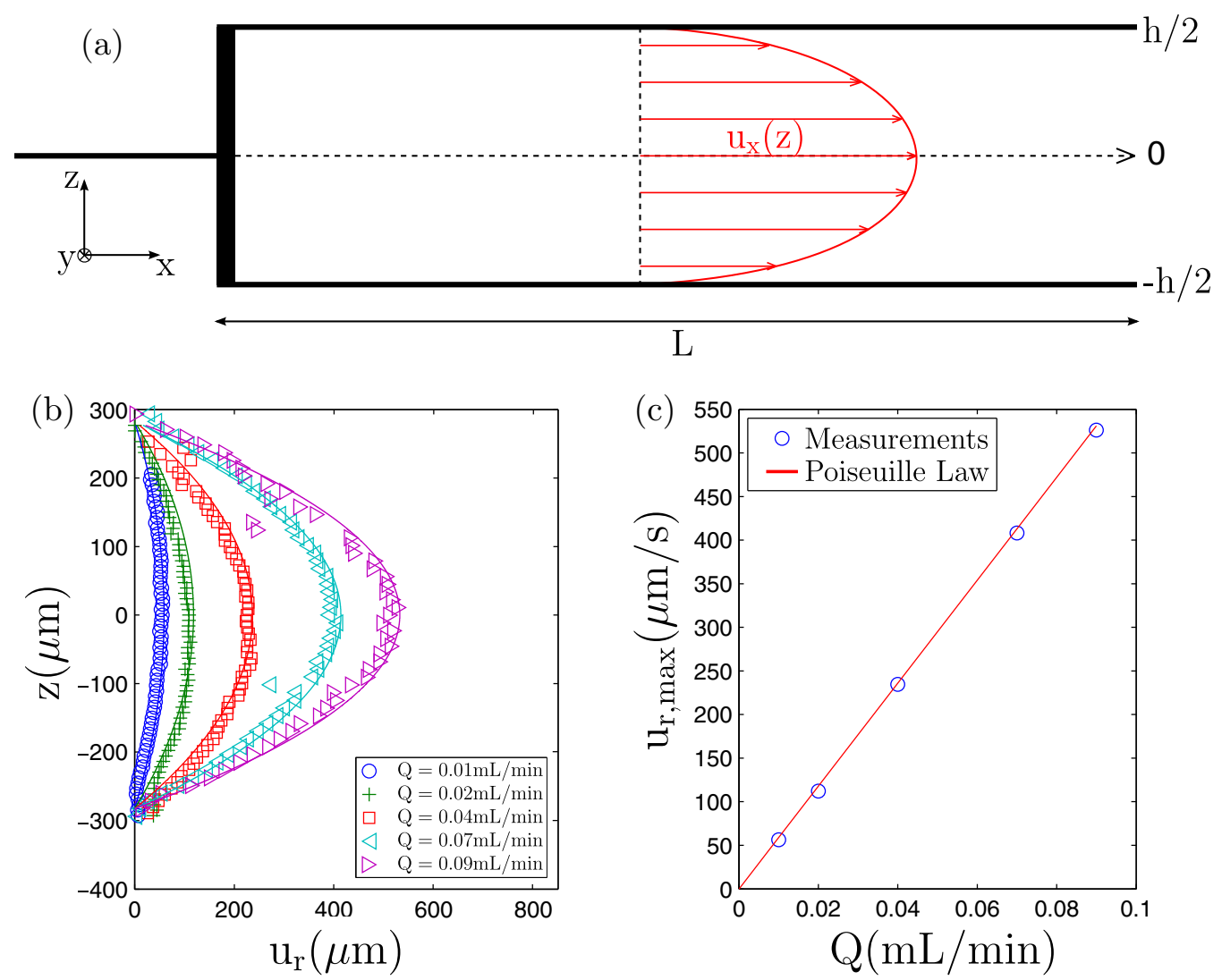

FIG. 9. (a) Schematic of the microchannel (thickness $h=500 \mu \mathrm{m}$, width $w=7.5 \mathrm{~mm}$, and length $L=10 \mathrm{~cm}$ ) used for the validation of the PIV. (b) Comparison between velocity measurements (symbols) and the Poiseuille parabolic profile (lines) for several flow rates $Q$. (c) Variation of the maximum experimental velocity (symbol) with respect to the flow rate compared with the Poiseuille law (line).

\section{APPENDIX}

\section{Verification of the PIV technique}

To validate our PIV measurements, we considered the classical Poiseuille flow in a rectangular microchannel with a small aspect ratio section (thickness $h=500 \mu \mathrm{m}$, width $w=7.5 \mathrm{~mm}$, and length $L=10 \mathrm{~cm}$ ), represented in Fig. 9(a). We performed velocity measurements for several imposed flow rates $Q$ and compared them with the Poiseuille law in Fig. 9(b). This microchannel has been chosen to have a small aspect ratio, $h / w \simeq 7.10^{-2}$, in order to allow the use of the Poiseuille parabolic profile [26,27]:

$$
u=\frac{h^{2}}{2 \eta_{0}} \frac{\partial p}{\partial x}\left[\frac{1}{4}-\left(\frac{z}{h}\right)^{2}\right] .
$$

In this approximation, the flow rate is:

$$
Q=\quad u_{x}(z) d y d z=\frac{w h^{3}}{12 \eta_{0}}\left|\frac{\partial p}{\partial x}\right| .
$$

Consequently, the velocity can be written in terms of flow rate:

$$
u=6 \frac{Q}{w h}\left[\frac{1}{4}-\left(\frac{z}{h}\right)^{2}\right] .
$$

Figure 9(b) shows a very good agreement between the experimental and theoretical profiles with no adjustable parameter. We then plotted on Fig. 9(c) the variation of the maximum velocity with respect to the flow rate. No deviation between the experiment and the Poiseuille law is observed showing that flow velocities can be measured precisely.

Measurements of particle movement without laser heating give a mean velocity around $4 \mu \mathrm{m} / \mathrm{s}$. That induces a relative

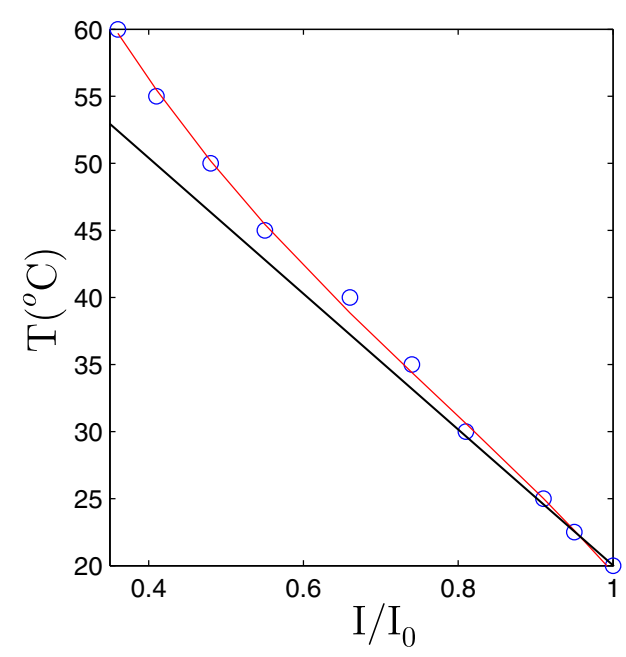

FIG. 10. Calibration curve for the fluorescence intensity of a solution of rhodamine-B versus temperature (empty circles) fitted with a polynome of degree 3 . Also represented is the approximated linear behavior expected close to the room temperature with a slope of $-0.5^{\circ} \mathrm{C} / \%$. 
(a)

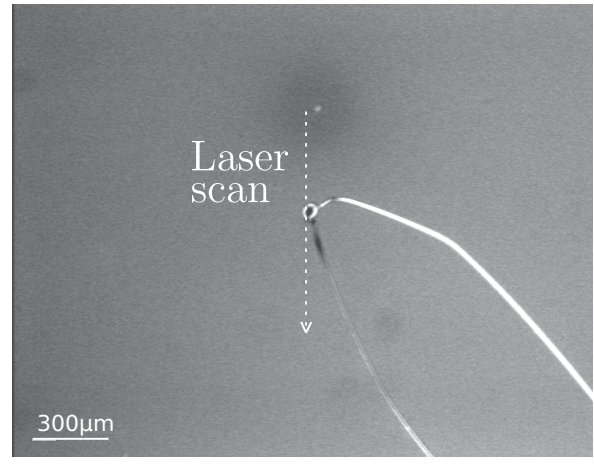

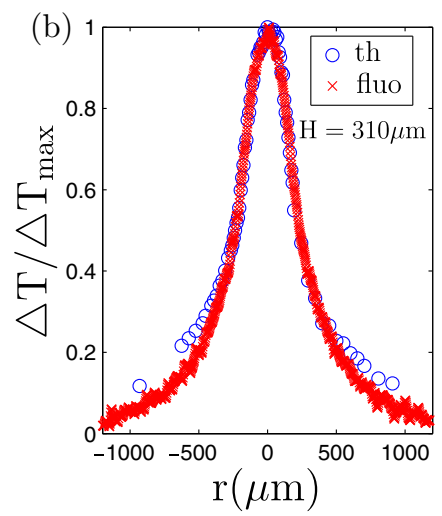



FIG. 11. (a) Picture of the miniaturized thermocouple (th) used for the measurements. (b), (c) Comparison of the temperature distribution deduced from the fluorescence (fluo) and the thermocouple (th) for $H=310 \mu \mathrm{m}$ (b) and $H=480 \mu \mathrm{m}$ (c).

error of $20 \%$ for a velocity $u=20 \mu \mathrm{m} / \mathrm{s}$ and down to $4 \%$ for $u=100 \mu \mathrm{m} / \mathrm{s}$ due to Brownian motion.

\section{Calibration curve for rhodamine-B}

Calibration of the fluorescence intensity was performed using a solution of $\mathrm{RhB}$ at the concentration $c=50 \mathrm{mg} / \mathrm{L}$ in a carbonate buffer (HEPES at $c=50 \mathrm{mg} / \mathrm{L}$ ) confined between two microscope slides. The slides were thermalized by placing on the top of the sample a water circulation controlled in temperature. A thermistance was used to measure the temperature inside the sample. For a given temperature, the fluorescence intensity $I(T)$ of the RhB solution is measured by averaging 20 successive pictures taken at $25 \mathrm{fps}$. To avoid any photobleaching process, the $\mathrm{RhB}$ solution is only exposed to the mercury lamp during image acquisition. The normalized fluorescence intensity defined as $I(T) / I\left(T=20^{\circ} \mathrm{C}\right)$ is then plotted versus temperature as shown in Fig. 10. For an accurate calibration, the curve is fitted with a polynome of degree 3 :

$T(r)=-66.8\left(\frac{I}{I_{0}}\right)^{3}+154.7\left(\frac{I}{I_{0}}\right)^{2}-173.7\left(\frac{I}{I_{0}}\right)+105.3$, where $I$ is the fluorescence intensity at a given temperature and $I_{0}$ is the fluorescence intensity at the room temperature. This trend is close to a linear behavior with a slope of $-0.5{ }^{\circ} \mathrm{C} / \%$ (or $2 \% /{ }^{\circ} \mathrm{C}$ for $I / I_{0}$ versus $T$ ) as reported previously [19].

\section{Comparison between thermocouple and fluorescence measurements}

To confirm the temperature measurements deduced from the fluorescence intensity of the rhodamine-B solution, we performed temperature measurements with a miniaturized thermocouple (Omega, type $\mathrm{K}$, diameter $\sim 50 \mu \mathrm{m}$ ) in a pure Millipore water solution (without rhodamine-B). Due to the fragility of the miniaturized thermocouple, the thermocouple is fixed in the sample while the laser is moved as indicated in Fig. 11(a). A picture of the experiment is also shown in Fig. 11(a), where a solution of RhB solution has been used for illustration purpose as fluorescence enables to detect much more easily the laser location. In Figs. 11(b) and 11(c), we compare the temperature distributions deduced from both the fluorescence intensity and the miniaturized thermocouple, respectively, for $H=310$ and $480 \mu \mathrm{m}$. These plots show a very nice superposition with a weak deviation on the


FIG. 12. Zoom on the maximum temperature elevation $\triangle T_{\max }$ with respect to the absorbed power $P_{\mathrm{abs}}$ for $H=185 \mu \mathrm{m}$ (a), $H=$ $310 \mu \mathrm{m}(\mathrm{b})$, and $H=480 \mu \mathrm{m}(\mathrm{c})$. 
wings between the two types of characterization, which validates the temperature measurements performed in this work.

\section{Linear behavior of $\Delta T_{\max }$ with $P_{\text {abs }}$}

In this Appendix we focus on the linear behavior of the maximum temperature elevation $\triangle T_{\max }$ with the absorbed power $P_{\text {abs }}$ described in Sec. II B, Fig. 3(a). We then plotted separately on Fig. 12 the variation $\triangle T_{\max }$ with $P_{\text {abs }}$ at low powers for each thickness. We added on this figure the uncertainties on temperature measurements deduced from the Lorentzian fit. The linear behavior at small $P_{\mathrm{abs}}$ appears clearly for the three investegated thicknesses. We found $\Delta T_{\max }=$ $1.9 \pm 0.3{ }^{\circ} \mathrm{C} / \mathrm{mW}$ for $H=185 \mu \mathrm{m}(\mathrm{a}), 1.5 \pm 0.2{ }^{\circ} \mathrm{C} / \mathrm{mW}$ for $H=310 \mu \mathrm{m}$ (b) and $1.6 \pm 0.2^{\circ} \mathrm{C} / \mathrm{mW}$ for $H=480 \mu \mathrm{m}$ (c).
[1] H. Bénard, J. Phys. Theor. Appl. 10, 254 (1901).

[2] L. Rayleigh, London, Edinburgh, Dublin Philos. Mag. J. Sci. 32 529 (1916).

[3] R. V. Birikh, J. Appl. Mech. Tech. Phys. 7, 43 (1966).

[4] F. M. Weinert and D. Braun, Nano Lett. 9, 4264 (2009).

[5] D. E. Cormack, L. G. Leal, and J. Imberger, J. Fluid Mech. 65, 209 (1974)

[6] D. E. Cormack, L. G. Leal, and J. H. Seinfeld, J. Fluid Mech. 65, 231 (1974)

[7] J. Imberger, J. Fluid Mech. 65, 247 (1974).

[8] D. Braun and A. Libchaber, Phys. Rev. Lett. 89, 188103 (2002).

[9] S. Fayolle, T. Bickel, and A. Würger, Phys. Rev. E 77, 041404 (2008).

[10] H. Chraïbi and J.-P. Delville, Phys. Fluids 24, 032102 (2012).

[11] A. Cazabat, F. Heslot, S. Troian, and P. Carles, Nature 346, 824 (1990).

[12] A. Kirdyashkin, Int. J. Heat Mass Transf. 27, 1205 (1984).

[13] A. Ortiz-Pérez and L. Dávalos-Orozco, Phys. Fluids 23, 084107 (2011).

[14] M. L. Cordero, E. Verneuil, F. Gallaire, and C. N. Baroud, Phys. Rev. E 79, 011201 (2009).
[15] M. Robert de Saint Vincent and J.-P. Delville, Phys. Rev. E 85, 026310 (2012).

[16] J. A. Vieyra Salas, J. M. van der Veen, J. J. Michels, and A. A. Darhuber, J. Phys. Chem. C 116, 12038 (2012).

[17] R. D. Keane and R. J. Adrian, Appl. Sci. Res. 49, 191 (1992).

[18] B. Selva, L. Daubersies, and J.-B. Salmon, Phys. Rev. Lett. 108, 198303 (2012).

[19] J. Sakakibara and R. Adrian, Exp. Fluids 26, 7 (1999).

[20] L. Kempers, J. Chem. Phys. 90, 6541 (1989).

[21] D. D. Gray and A. Giorgini, Int. J. Heat Mass Transf. 19, 545 (1976).

[22] R. Rusconi, L. Isa, and R. Piazza, JOSA B 21, 605 (2004).

[23] H. Chraibi, J. Petit, R. Wunenburger, and J.-P. Delville, Phys. Rev. Lett. 111, 044502 (2013).

[24] H. Chraibi, R. Wunenburger, D. Lasseux, J. Petit, J.-P. Delville et al., J. Fluid Mech. 688, 195 (2011).

[25] C. Vest, Phys. Fluids 17, 1945 (1974).

[26] H. A. Stone, A. D. Stroock, and A. Ajdari, Annu. Rev. Fluid Mech. 36, 381 (2004).

[27] X. Zheng and Z.-h. Silber-Li, Exp. Fluids 44, 951 (2008). 